\title{
Cystoscopic Balloon Dilatation: A Novel Tool in The Armamentarium to Deal with Complex Intra Hepatic Biliary Strictures
}

\author{
Gaurav Aggarwal ${ }^{1 *}$, Sujoy Gupta ${ }^{1}$, Sudeep Banerjee², V Sitaram² \\ ${ }^{1}$ Department of Urological-Oncology, Tata Medical Center, Kolkata, India \\ ${ }^{2}$ Department of Gastrointestinal-Oncology, Tata Medical Center, Kolkata, India
}

*Corresponding author: Gaurav Aggarwal, Department of Urological-Oncology, Tata Medical Center, Kolkata-700156, India. Tel: +91-07873947779; Email: drgaurav1981@rediffmail.com/gaurav.aggarwal@tmckolkata.com

Citation: Aggarwal G, Gupta S, Banerjee S, Sitaram V (2019) Cystoscopic Balloon Dilatation: A Novel Tool in The Armamentarium to Deal with Complex Intra Hepatic Biliary Strictures. J Surg 13: 1237 DOI: 10.29011/2575-9760.001237

Received Date: 04 July, 2019; Accepted Date: 19 July, 2019; Published Date: 24 July, 2019

\begin{abstract}
Biliary strictures commonly involve the extrahepatic biliary system. Strictures that involve the segmental intrahepatic ducts, are rarely seen and equally difficult to treat. They may often be detected incidentally after imaging performed for other reasons. Their onset is often a prelude to cholestasis, cholangitis and sepsis, if left untreated. Endourological advancements in technology, equipment as well as surgical-expertise have laid open a new corridor for the role of the urologist, outside of the normal urinary tract. We present the case of a 29 -year-old lady, wherein with urological expertise, a cystoscopic balloon dilatation of the strictured junction of the right anterior and posterior sectoral ducts was managed, following failure of conventional treatment modalities, thus highlighting an innovative tool in the management armamentarium of complex intra hepatic biliary strictures.
\end{abstract}

Keywords: Bile duct strictures; Cystoscopic balloon dilatation; Endourology; Intrahepatic biliary strictures

\section{Introduction}

Biliary strictures commonly involve the extrahepatic biliary system. Strictures that involve the segmental intrahepatic ducts, are much rarer and equally difficult to treat [1]. They may often be detected as incidental findings after imaging performed for other reasons [2]. Though a low reported incidence, their onset is a prelude to cholestasis, cholangitis and sepsis, if left untreated [3]. Endourological advancements in technology, equipment as well as surgical-expertise have laid open a new corridor for the role of the urologist, outside of the normal urinary tract. We present the case of a 29-year-old lady, wherein with urological expertise, a cystoscopic balloon dilatation of the strictured junction of the right anterior and posterior sectoral ducts was managed, following failure of conventional treatment modalities, thus highlighting a novel tool in the management armamentarium of complex intra hepatic biliary strictures.

Case

A 29-year-old lady presented to our clinic with acute abdominal pain, associated with fever and chills. She had undergone a cholecystectomy with common bile duct (CBD) excision and a roux-en-Y hepaticojejunostomy (HJ) in 2006, with a differential of choledochal cyst or type IV Mirizzi's syndrome. She was found to have a strictured HJ, which was revised. She was asymptomatic for about a year thereafter, but again presented with persistent vague upper abdominal pain, intermittent fever \& vomiting episodes. An MRI abdomen was done which showed a stricture at the insertion of the right posterior sectoral $\&$ right anterior sectoral duct with a filling defect in the lateral segment ducts with upstream dilatation (Figure 1). 


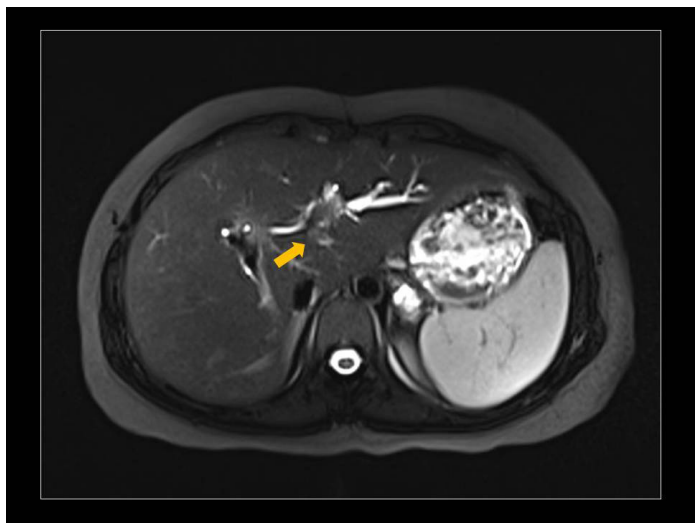

Figure 1: MRI abdomen showing a stricture at the insertion of the right posterior sectoral \& right anterior sectoral duct (arrow head) with a filling defect in the lateral segment ducts with upstream dilatation.

An ERCP was attempted but a guide wire couldn't be passed into the right duct. She was subsequently taken up for exploration. Intra-operatively, using a 22 Fr cystoscope (passed in via an enterotomy on the anterior jejuna wall), a 0.035 -inch guide wire was negotiated across the strictured segment (under image intensifier guidance) (Figure 2).

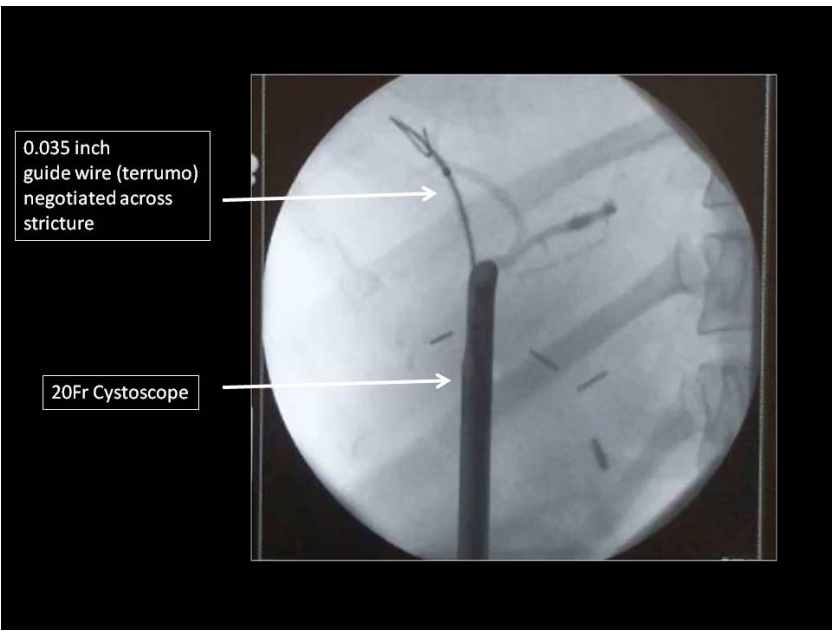

Figure 2: Shows the 0.035-inch guide wire negotiated across the strictured right intrahepatic duct segment.

A balloon dilator (routinely used in urology for ureteric orifice/stricture dilatation) was passed over the guide wire and with 10 atmosphere pressure, complete dilatation of the structured segment was achieved (Figure 3). The patient was asymptomatic thereafter and has been doing well 3 years since.

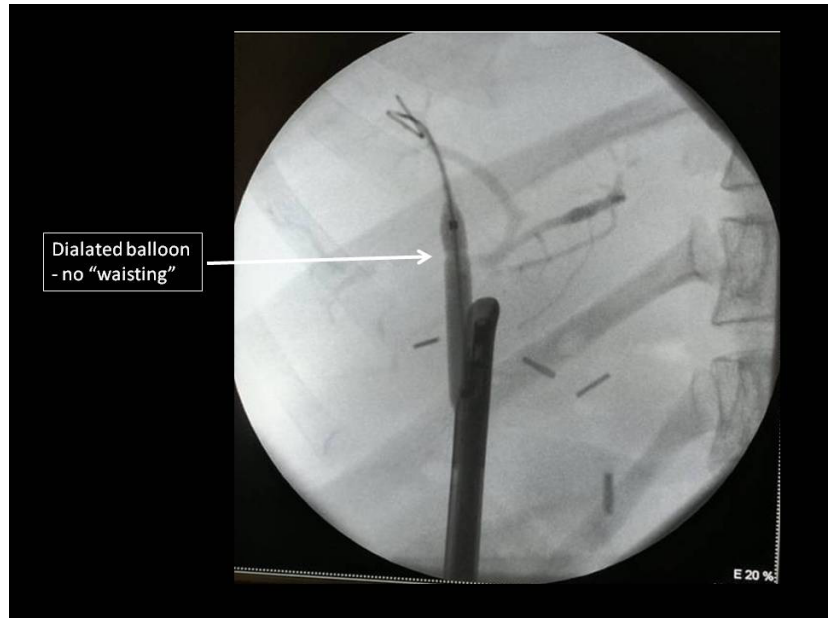

Figure 3: Complete dilatation of the stricture, with no "Waisting" of the balloon.

\section{Discussion}

Strictures of the biliary tract commonly involve the extrahepatic bile ducts. Those involving the segmental intrahepatic ducts are much rarer and equally difficult to treat, with no consensus as to the best therapeutic approach [1]. Focal intrahepatic strictures, often associated with hepatolithiasis, may often be detected incidentally as well, after imaging performed for other reasons [2]. Though a low reported incidence, their onset may predispose to cholestasis, cholangitis and sepsis, if not adequately treated [3]. Conventional approaches such as percutaneous balloon dilatation, Endoscopic Retrograde Cholangiopancreatography (ERCP) and Percutaneous Transhepatic Biliary Drainage (PTBD) have been reported in literature with success rates varying from 93-100\% $[4,5]$. The therapeutic conundrum for intra hepatic biliary strictures often mandates a multi-disciplinary approach, especially when conventional modalities fail, due to anatomo-pathological factors or otherwise. With the advent of minimally invasive urology, hybrid approaches to the intra-hepatic biliary system have begun gaining momentum [6]. There are reports in literature about the use of semi rigid/ flexible ureteroscopy and laser/pneumatic/electrohydraulic lithotripsy of intrahepatic biliary calculi $[7,8]$.

To the best of our knowledge, ours could be an index case where cystoscopic balloon dilatation was successful in treating a complex intra hepatic biliary ductal stricture, with no symptom advent till date, 3 years post procedure. Thus, cystoscopic balloon dilatation may be a new cog in the wheel for treatment of intra hepatic strictures, not amenable to conventional treatments. 


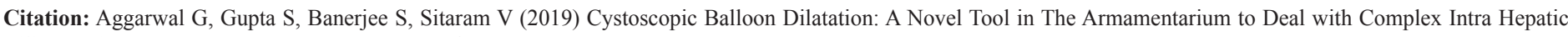
Biliary Strictures. J Surg 13: 1237 DOI: 10.29011/2575-9760.001237

\section{Conclusion}

With advancements in endourology, urological expertise has become sacrosanct even in non-urological procedures. An "out of the box" approach is often mandated, when standard methodology falls short of expectations. A multi-disciplinary approach with endourological expertise should hence be considered, especially in complex endo-biliary conditions. Thus cystoscopic balloon dilatation is an innovative cross-discipline tool in the management armamentarium of complex intra hepatic biliary strictures.

\section{References}

1. Mercadier M, Fingerhut A (1984) Strictures of the intrahepatic bile ducts. World J Surg 8: 15-21.

2. Kim HJ, Lee KT, Kim SH, Lee JK, Lim JH, et al. (2003) Differential diagnosis of intrahepatic bile duct dilatation without demonstrable mass on ultrasonography or CT: benign versus malignancy. J Gastroenterol Hepatol 18: 1287-1292.

3. Maggi U, Paone G, Lauro R, Fornoni G, Chierici A, et al. (2016) Holmium intraductal laser lithotripsy of biliary stones in liver grafts. Transplantation Proceedings 48: 380-382.
4. Kocher M, Cerna M, Havlík R, Kral V, Gryga A, et al. (2007) Percutaneous treatment of benign bile duct strictures. European journal of radiology 62 : 170-174.

5. Thomas R, Köcher M, Cerna M, Kozak J, Burval S, et al. (2009) Is the biliary manometric perfusion test effective in the evaluation of balloon dilatation treatment success of benign biliary strictures?. Biomed Papers 153: 153-156.

6. Nadler RB, Rubeinstein JN, Kim SC, Weiser AC, Lohr MN, et al. (2002) Percutaneous Hepatolithotomy, The northwestern experience. J Endourology 16: 293297.

7. Rajaian S, Mukherjee A, Gopalakrishnan G (2006) A Novel Use of Semi Rigid Ureteroscope for a Non Urological A Point of Technique. Kuwait Medical Journal 38: 56-58.

8. Sninsky BC, Sehgal PD, Hinshaw JL, McDermott JC, Nakada SY (2014) Expanding endourology for biliary stone disease: the efficacy of intracorporeal lithotripsy on refractory biliary calculi. J Endourol 28: 877-880. 